\title{
Fine-scale evolution: genomic, phenotypic and ecological differentiation in two coexisting Salinibacter ruber strains
}

\author{
Arantxa Peña ${ }^{1}$, Hanno Teeling ${ }^{2}$, Jaime Huerta-Cepas ${ }^{3,4,10}$, Fernando Santos ${ }^{1}$, Pablo Yarza ${ }^{5}$, \\ Jocelyn Brito-Echeverría ${ }^{5}$, Marianna Lucio ${ }^{6}$, Philippe Schmitt-Kopplin ${ }^{6}$, \\ Inmaculada Meseguer ${ }^{7}$, Chantal Schenowitz ${ }^{8}$, Carole Dossat ${ }^{8}$, Valerie Barbe ${ }^{8}$, \\ Joaquín Dopazo ${ }^{3,4}$, Ramon Rosselló-Mora ${ }^{5}$, Margarete Schüler ${ }^{2}$, Frank Oliver Glöckner ${ }^{2,9}$, \\ Rudolf Amann ${ }^{2}$, Toni Gabaldón ${ }^{3,10}$ and Josefa Antón ${ }^{1}$ \\ ${ }^{1}$ Departamento de Fisiología, Genética y Microbiología, and IMEM, Universidad de Alicante, Apartado 99, \\ Alicante, Spain; ${ }^{2}$ Max Planck Institute for Marine Microbiology, Celsiusstrasse 1, Bremen, Germany; \\ ${ }^{3}$ Department of Bioinformatics, Centro de Investigación Príncipe Felipe, Autopista del Saler 16, Valencia, \\ Spain; ${ }^{4}$ Functional Genomics Node (INB), Centro de Investigación Príncipe Felipe (CIPF), Valencia, Spain; \\ ${ }^{5}$ Marine Microbiology Group, Institut Mediterrani d'Estudis Avançats, Esporles, Spain; ${ }^{6}$ Helmholtz Zentrum \\ Muenchen, German Research Center for Environmental Health, Institute of Ecological Chemistry, \\ Neuherberg, Germany; ${ }^{7}$ Departamento de Producción Vegetal y Microbiología, Universidad Miguel \\ Hernández. Avda. de la Universidad s/n., Elche (Alicante), Spain; ${ }^{8} C E A / D S V / I G / G e n o s c o p e$, Evry, France \\ and ${ }^{9}$ Jacobs University Bremen gGmbH, Campusring 1, Bremen, Germany
}

\begin{abstract}
Genomic and metagenomic data indicate a high degree of genomic variation within microbial populations, although the ecological and evolutive meaning of this microdiversity remains unknown. Microevolution analyses, including genomic and experimental approaches, are so far very scarce for non-pathogenic bacteria. In this study, we compare the genomes, metabolomes and selected ecological traits of the strains M8 and M31 of the hyperhalophilic bacterium Salinibacter ruber that contain ribosomal RNA (rRNA) gene and intergenic regions that are identical in sequence and were simultaneously isolated from a Mediterranean solar saltern. Comparative analyses indicate that $S$. ruber genomes present a mosaic structure with conserved and hypervariable regions (HVRs). The HVRs or genomic islands, are enriched in transposases, genes related to surface properties, strain-specific genes and highly divergent orthologous. However, the many indels outside the HVRs indicate that genome plasticity extends beyond them. Overall, $10 \%$ of the genes encoded in the M8 genome are absent from M31 and could stem from recent acquisitions. S. ruber genomes also harbor 34 genes located outside HVRs that are transcribed during standard growth and probably derive from lateral gene transfers with Archaea preceding the M8/M31 divergence. Metabolomic analyses, phage susceptibility and competition experiments indicate that these genomic differences cannot be considered neutral from an ecological perspective. The results point to the avoidance of competition by micro-niche adaptation and response to viral predation as putative major forces that drive microevolution within these Salinibacter strains. In addition, this work highlights the extent of bacterial functional diversity and environmental adaptation, beyond the resolution of the 16S rRNA and internal transcribed spacers regions.
\end{abstract}

The ISME Journal (2010) 4, 882-895; doi:10.1038/ismej.2010.6; published online 18 February 2010

Subject Category: evolutionary genetics

Keywords: comparative genomics; microdiversity; micro-evolution; phage; Salinibacter

Correspondence: J Antón, Departamento de Fisiología, Genética y Microbiología, Universidad de Alicante, División de Microbiología, Alicante 03080, Spain.

E-mail: anton@ua.es

${ }^{10}$ Current address: Bioinformatics and Genomics Programme, Center for Genomic Regulation (CRG), Dr Aiguader, 88, Barcelona 08003, Spain.

Received 28 October 2009; revised 4 January 2010; accepted 5 January 2010; published online 18 February 2010

\section{Introduction}

Microbial genomics has undergone an amazing development since the finishing of the first bacterial genome in 1995 (Fleischmann et al., 1995). Although pathogens and extremophiles with biotechnological potential were initially at focus, recently attention has shifted to environmentally relevant microorganisms that constitute the majority of the existing microbial biodiversity. Marine habitats, covering 
more than $70 \%$ of our planet's surface, have gained special attention in microbial genome sequencing, as illustrated by more than 200 available draft genomes contributed by the Marine Microbiology Initiative of the Gordon and Betty Moore foundation (http:// www.moore.org).

The possibility of comparing complete genomes of related prokaryotes provides unique opportunities to study the course of evolution at the DNA level. Although comparisons of genomes of different species give insights on a larger scale of evolution, the so-called microevolution (Abby and Daubin, 2007) can only be addressed by comparing genomes of different strains within the same species. Microevolution studies can reveal how bacterial genomes evolve, provide insights in the hidden diversity within a species' population and elucidate the ecological mechanisms that drive microbial radiation (Sikorski, 2008). Current knowledge on microevolution stems mostly from studying strains of pathogenic bacteria but is unmatched for environmentally relevant prokaryotes. So far, comparative studies on genomic adaptations of environmentally relevant prokaryotes have focused on ecotypes, that is, populations of the same species with distinct ecological strategies (Cohan and Koeppel, 2008). Examples are studies on Prochlorococcus sp. (Coleman et al., 2006) or Alteromonas macleodii (Ivars-Martínez et al., 2008) isolates, which have ecotypes that are also distinguishable through their $16 \mathrm{~S}$ ribosomal RNA (rRNA) gene(s) or 16S-23S rRNA internal transcribed spacers (ITS). These ecotypes, however, would not fall within the 16S rRNA gene microdiversity clusters ( $>99 \%$ similarity) described by Acinas et al. (2004) for coastal bacterioplankton. These clusters have been subsequently described for other environments, including hypersaline systems, and have been corroborated by metagenomic data (Wilmes et al., 2008). The widespread presence of this microdiversity within microbial populations raises three main questions: (i) How much functional and genomic diversity is hidden behind this diversity? (ii) What is the ecological meaning of this microdiversity? and (iii) How does this microdiversity arise?

In few cases these questions have been answered. For instance, Vibrio splendidus, which is composed of strains that are $99 \%$ identical in $16 \mathrm{~S}$ rRNA gene sequences and shows a wide genomic diversity (Thomson et al., 2005), differentiates into 15 microdiverse habitat-associated clusters, possibly by invading new niches or partitioning resources at increasingly fine scales (Hunt et al., 2008). In other cases, response to viral predation and neutral diversification have been suggested as mechanisms for generating microdiversity (Wilmes et al., 2008). In any case, comparisons of both genomes and putative ecological traits for very closely related strains are so far very scare (for examples, see Wilmes et al. (2008) and below in this paper).
The genus Salinibacter can be found in hypersaline environments worldwide. Salinibacter populations have been detected by culture and molecular approaches in crystallizer ponds and salt lakes in places as distant as Australia, the Peruvian Andes, Turkey and Spain (Antón et al., 2008). These hypersaline environments harbor dense communities of extremely halophilic Archaea, often dominated by the square-shaped archaeon Haloquadratum walsbyi. Nevertheless, Salinibacter ruber can account for a large part of the respective microbial communities, with population sizes ranging up to more than $10^{7}$ cells $\mathrm{ml}^{-1}$ (around $30 \%$ of total cell counts). The description of the species $S$. ruber was based on five strains (Antón et al., 2002), of which two (M8 and M31, see below) were simultaneously isolated in September 1999 from two samples taken from the same crystallizer pond of a coastal solar saltern in Mallorca (Balearic Islands, Spain). A multi-locus sequencing analysis among $10 \mathrm{~S}$. ruber strains, including M8 and M31, from salterns in three distant locations (Atlantic, Andean and Mediterranean) could not show a genetic divergence related to the geographical origin of the isolates (Rosselló-Mora et al., 2008). Phylogenetic reconstructions using these selected genes resulted in different tree topologies, a phenomenon that has been previously observed in extremely halophilic Archaea from solar salterns (Papke et al., 2004) and is considered to be an indication for high intra-specific recombination rates (Feil, 2004). Consistent with this, a split decomposition (Huson and Bryant, 2006) of the concatenated sequences of the genes provided statistically significant evidence for recombination among these 10 $S$. ruber strains (Antón et al., unpublished results).

Strains M8 and M31 share an identical rRNA operon sequence, including identical ITS. Among all the isolated $S$. ruber strains M8 and M31 are among the most closely related (based on rRNA, Pulsed Fiel Gel Electroforesis and Random Amplification of Polymorphic DNA analysis) (Peña et al., 2005; Antón et al., 2008), and would thus represent an example of very short-scale microdiversity within this species. However, their level of DNA-DNA hybridization is around $88 \%$, which indicates that they have noteworthy genomic differences (Peña et al., 2005). These differences are intriguing given that they inhabit a rather homogenous environment that at first glance offers little opportunities for micro-niche differentiations.

The key questions are: what are the genomic differences among these strains?, how did they arise and to which extent are they neutral or have an ecological relevance? For this purpose, the genome of strain M8 has been fully sequenced and compared with the previously sequenced M31 genome (type strain DSMZ $13855^{\mathrm{T}}$, Mongodin et al., 2005). Phenotypic differences between both strains were examined by metabolomic analysis using Fourier transform ion cyclotron resonance mass spectrometry (Rosselló-Mora et al., 2008). To explore their 
putative ecological differences in nature, their phage susceptibility against different saltern waters was assayed, and competition experiments between both strains carried out. In terms of microevolution, this is the shortest-scale study on genomic changes and adaptations with representatives of the same species, beyond the ecotype level, conducted so far.

\section{Materials and methods}

Architecture and analysis of M8 genome sequence Potential protein coding open reading frames were identified with mORFind v2 (Waldmann and Teeling, unpublished) (Supplementay Material). The sequences have been submitted to EMBL database (http://www. ebi.ac.uk/embl) (accession nos. FP565814 (chromosome) and FP565810 (plasmid pSR11), FP565811 (pSR56), FP565812 (pSR61) and FP565813 (pSR84)).

Phylome reconstruction and orthology determination We reconstructed a complete set of phylogenetic trees, also known as phylome, for the two $S$. ruber genomes. All trees and aligments can be accessed through www.phylomedb.org (Huerta-Cepas et al., 2008) (Supplementary Figure 1). Orthologous and paralogous relationships among genes encoded in the two Salinibacter genomes were determined through a phylogenetic approach. (Supplementay Material).

Identification of genes putatively involved in BacteriaArchaea interdomain lateral gene transfer (LGT)

We adopted a strategy of combining phylogeny and oligonucleotide frequency analyses to identify genes putatively involved in interdomain LGT events (Supplementay Material).

\section{Metabolomic analysis}

Metabolite profiles for extracellular and pellet fractions of pure cultures of M8 and M31 were analyzed by Fourier transform ion cyclotron resonance mass spectrometry (FT-ICMS) (Supplementay Material).

\section{Competition experiments between M8 and M31 and} phage susceptibility

Co-cultures of M8 and M31 were monitored by realtime PCR using strain-specific primers (Supplementay Material). Phage susceptibility for each strain was monitored by the plaque-assay technique as described in Supplementay Material.

\section{Results and discussion}

Genomic differences among M8 and M31 strains: plasticity and mosaic structure

Overall comparison between M8 and M31 genomes. The complete genome sequence of strain M8 was determined, analyzed and compared with the previously published M31 genome (Mongodin et al., 2005). The main results from these analyses are summarized in Table 1 and Figures 1 and 2 .

With the exception of hypervariable regions or genomic islands (HVRs, described in detail below), high levels of conservation in gene order and orientation could be observed between M8 and M31 chromosomes (see dot-plot in Supplementary Figure 2). Such degree of synteny is expected because of the phylogenetic proximity of the two strains. However, 518 (18\%) of the M8/M31 orthologous gene pairs had rather low levels of amino acid sequence identities, varying on a scale from $23 \%$ to $90 \%$. In general, these divergent orthologs were evenly distributed throughout the whole genome (Figure 2, fourth circle inward; Figure 1), although the most divergent genes were concentrated in HVRs (see below).

Overall, M8 and M31 share around 90\% of their genes or, in other words, there are $10 \%$ strainspecific genes. Among the shared genes, 17\% had no homologs in public repositories and should thus for now be considered as species specific. The species-specific genes would be part of the $S$. ruber core genome (Feil, 2004), while the strain-specific genes are part of the species' accessory genome (Figure 3). Together, core and accessory genomes will be part of the species pan-genome (Meddini et al., 2005). Although most strain-specific genes were concentrated in HVRs, many gene insertions and/or deletions and transpositions could be observed also in aligned (that is, non-HVR) areas of both chromosomes, which, together with the 'divergent' genes (see above) accounted for a considerable part of the differences outside the HVRs. The remarkable presence of indels outside hypervariable regions has been previously described for Pelagibacter ubique isolates. Whilhelm et al. (2007) compared the genomes of two $P$. ubique isolates from the Oregon coast (one base difference in their $16 \mathrm{~S}$ rRNA genes; 98\% ITS identity; genome size of approximately $1.3 \mathrm{Mb}$ ) and found 62 gene indels outside HVRs. The investigators suggested that these indels could be polymorphisms, similar to allelic polymorphisms. The number of such indels in $S$. ruber $\mathrm{M} 8$ is 166, which is similar to that of $P$. ubique when normalized for the chromosome size (45.9 versus $47.7 \mathrm{Mb}^{-1}$ ).

The identified orthologous gene pairs exhibited an average amino acid identity of $94.2 \%$ and an average nucleotide identity (ANI) of 93.5\%. This ANI value is lower than that derived from random fragment blast pairs (ANIb, 98.5\%), which can be explained by the different thresholds used in their calculations. In this regard, the level of nucleotide identity between orthologous pairs reached 98.4 when indels were not considered (exact matches over aligned residues), a level that is more comparable to that of ANIb. It is also reasonable to assume that an ANI calculated with orthologs reflects existing evolutionary differences more accurately 
Table 1 Comparison of Salinibacter ruber DSM 13588 general features with S. ruber strain M8

\begin{tabular}{|c|c|c|c|c|c|c|}
\hline & \multicolumn{6}{|c|}{ S. ruber } \\
\hline & \multicolumn{4}{|c|}{ M8 } & \multicolumn{2}{|c|}{ M31 DSM 13588} \\
\hline \multicolumn{7}{|l|}{ Chromosome } \\
\hline Length (pb) & \multicolumn{4}{|c|}{3619447} & \multicolumn{2}{|r|}{3551823} \\
\hline $\mathrm{G}+\mathrm{C}$ content & \multicolumn{4}{|c|}{$66.12 \%$} & \multicolumn{2}{|r|}{$66.29 \%$} \\
\hline ORF number & \multirow{2}{*}{\multicolumn{4}{|c|}{3086}} & \multicolumn{2}{|r|}{2934} \\
\hline rRNAs & \multicolumn{2}{|c|}{3} & & & \multicolumn{2}{|r|}{3} \\
\hline tRNAs & \multicolumn{4}{|c|}{43} & \multicolumn{2}{|r|}{44} \\
\hline Total aligned sites: & \multicolumn{4}{|c|}{3348702} & \multirow{2}{*}{\multicolumn{2}{|c|}{$\begin{array}{c}3283845 \\
\text { nd }\end{array}$}} \\
\hline$\%$ Total ANI & & & & & & \\
\hline$\%$ similarity (аa) & & & & & & \\
\hline$\%$ similarity (nt) & & & & & & \\
\hline Total orthologous ${ }^{\mathrm{a}}$ & & & & & & \\
\hline COG categories $^{\mathrm{b}}$ & & & $\%$ & otal & No & $\%$ of total \\
\hline J-Translation, ribosomal structure and biogenesis & & & & & 106 & 3.69 \\
\hline K-Transcription & & & & & 110 & 3.83 \\
\hline L-DNA replication, recombination and repair & & & & & 145 & 5.05 \\
\hline D-Cell division and chromosome partition & & & & & 23 & 0.80 \\
\hline V-Defense mechanisms & & & & & 47 & 1.64 \\
\hline O-Posttranslational modification, protein turnover, chaperones & & & & & 85 & 2.96 \\
\hline M-Cell envelope biogenesis, outer membrane & & & & & 147 & 5.12 \\
\hline N-Cell motility and secretion & & & & & 57 & 1.99 \\
\hline U-Intracellular trafficking, secretion, and vesicular transport & & & & & 51 & 1.78 \\
\hline T-Signal transduction mechanisms & & & & & 141 & 4.91 \\
\hline C-Energy production and conversion & & & & & 123 & 4.29 \\
\hline G-Carbohydrate transport and metabolism & & & & & 86 & 3.00 \\
\hline E-Amino acid transport and metabolism & & & & & 182 & 6.34 \\
\hline P-Inorganic ion transport and metabolism & & & & & 117 & 4.08 \\
\hline F-Nucleotide transport and metabolism & & & & & 60 & 2.10 \\
\hline H-Coenzyme metabolism & & & & & 83 & 2.89 \\
\hline I-Lipid metabolism & & & & & 72 & 2.51 \\
\hline Q-Secondary metabolites biosynthesis, transport and catabolism & & & & & 53 & 1.85 \\
\hline R-General function prediction only & & & & & 257 & 8.96 \\
\hline S-Function unknown & & & & & 132 & 4.60 \\
\hline No COG categories & & & & & 989 & $34.46 \%$ \\
\hline Conserved hypothetical protein & & & & & 320 & 10.91 \\
\hline Hypothetical protein & & & & & 542 & 18.47 \\
\hline Plasmids & & & & & & 1 \\
\hline Name & pSR11 & pSR56 & pSR61 & pSR84 & & pSR35 \\
\hline Length (Mb) & 11.23 & 56.53 & 61.37 & 84.34 & & 35.5 \\
\hline $\mathrm{G}+\mathrm{C}$ content & 63.29 & 60.03 & 59.58 & 63.19 & & 59.70 \\
\hline ORF number & 13 & 38 & 50 & 70 & & 33 \\
\hline Total orthologous ${ }^{\mathrm{a}}$ & 1 & 11 & 16 & 28 & & nd \\
\hline COG categories $^{\mathrm{b}}$ & & & & & & \\
\hline K-Transcription & 0 & 1 & 0 & 2 & & 0 \\
\hline L-DNA replication, recombination and repair & 0 & 7 & 5 & 12 & & 6 \\
\hline D-Cell division and chromosome partition & 1 & 1 & 1 & 0 & & 1 \\
\hline V-Defense mechanisms & 0 & 4 & 0 & 0 & & 1 \\
\hline M-Cell envelope biogenesis, outer membrane & 0 & 0 & 1 & 12 & & 0 \\
\hline N-Cell motility and secretion & 0 & 0 & 0 & 2 & & 0 \\
\hline U-Intracellular trafficking, secretion, and vesicular transport & 0 & 0 & 1 & 0 & & 0 \\
\hline T-Signal transduction mechanisms & 0 & 0 & 0 & 7 & & 0 \\
\hline H-Coenzyme metabolism & 0 & 0 & 0 & 1 & & 0 \\
\hline E-Amino acid transport and metabolism & 0 & 1 & 0 & 0 & & 0 \\
\hline R-General function prediction only & 0 & 0 & 2 & 0 & & 1 \\
\hline S-Function unknown & 0 & 3 & 1 & 3 & & 1 \\
\hline No COG categories & & & & & & \\
\hline Hypothetical proteins & 9 & 14 & 30 & 25 & & 23 \\
\hline Conserved hypothetical proteins & 3 & 7 & 9 & 10 & & \\
\hline
\end{tabular}

Abbreviations: ANI, average nucleotide identity; COG, clusters of orthologous group; ORF, open reading frame; rRNA, ribosomal RNA tRNAs, transfer RNAs.

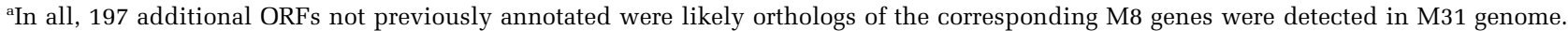

${ }^{\mathrm{b}}$ Many of the genes have assignment to more than one COG category. 

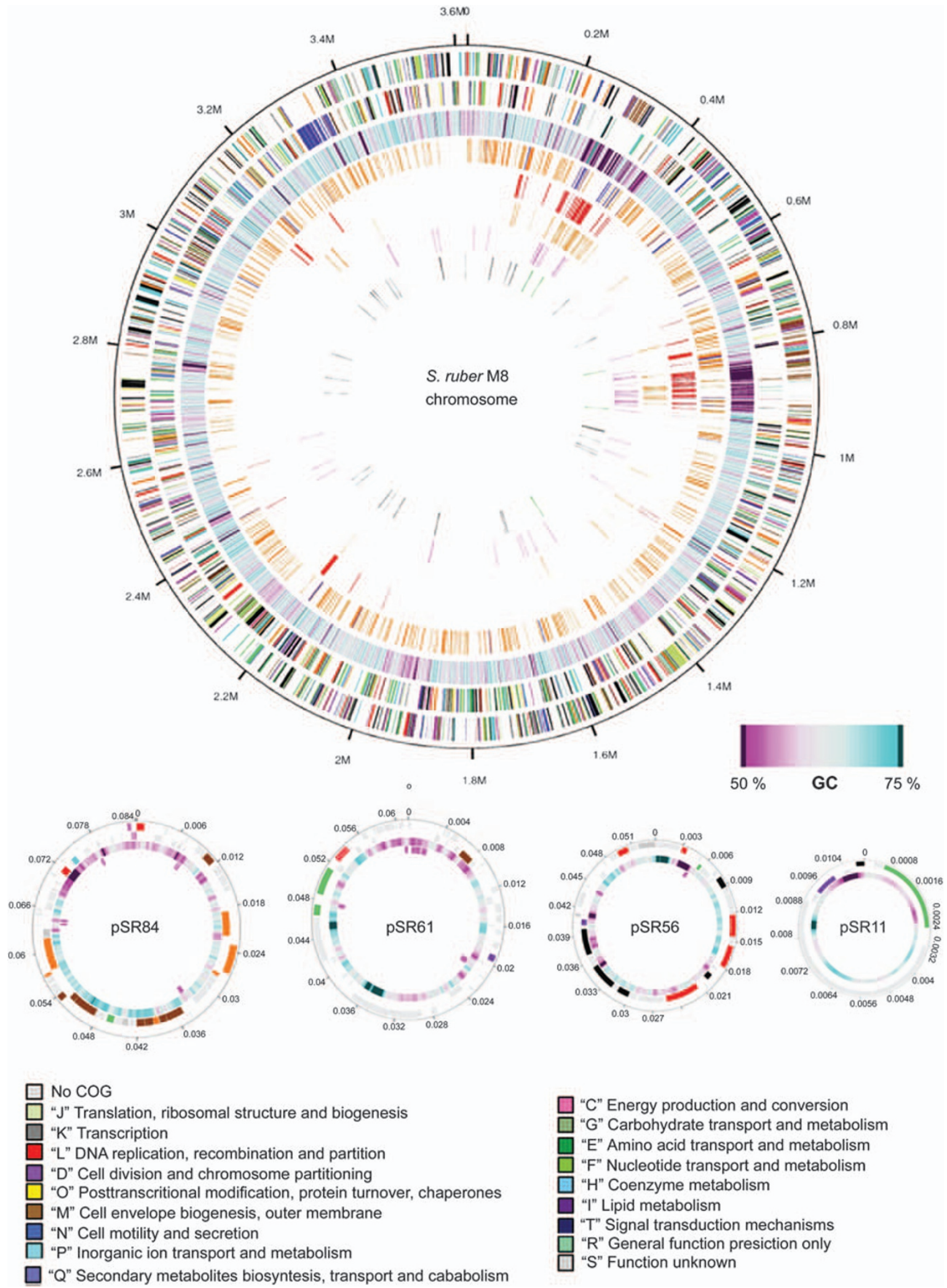

Figure 1 Circular representations of the M8 chromosome and its plasmids. For the chromosome, from the outside to inside: Circles 1-2: annotation by clusters of orthologous group (COG) functional categories (see insert). Circle 3: $\mathrm{G}+\mathrm{C}$ content. Circle 4: divergent genes in Salinibacter ruber M8/M31 marked in blue and orange the genes below 50\% or 90\% identity, respectively. Circle 5: genes present in M8 and some other organisms in the database but absent in M31. Circle 6: strain-specific genes in S. ruber (genes absent from M31 and databases). Circle 7: transposases. Circle 8: putative LGT from Archaea. For the plasmids, the outer and second circles represented open reading frames (ORFs) on the plus and minus strands, colored by COG functional categories. The third circle represents the $\mathrm{G}+\mathrm{C}$ content. 

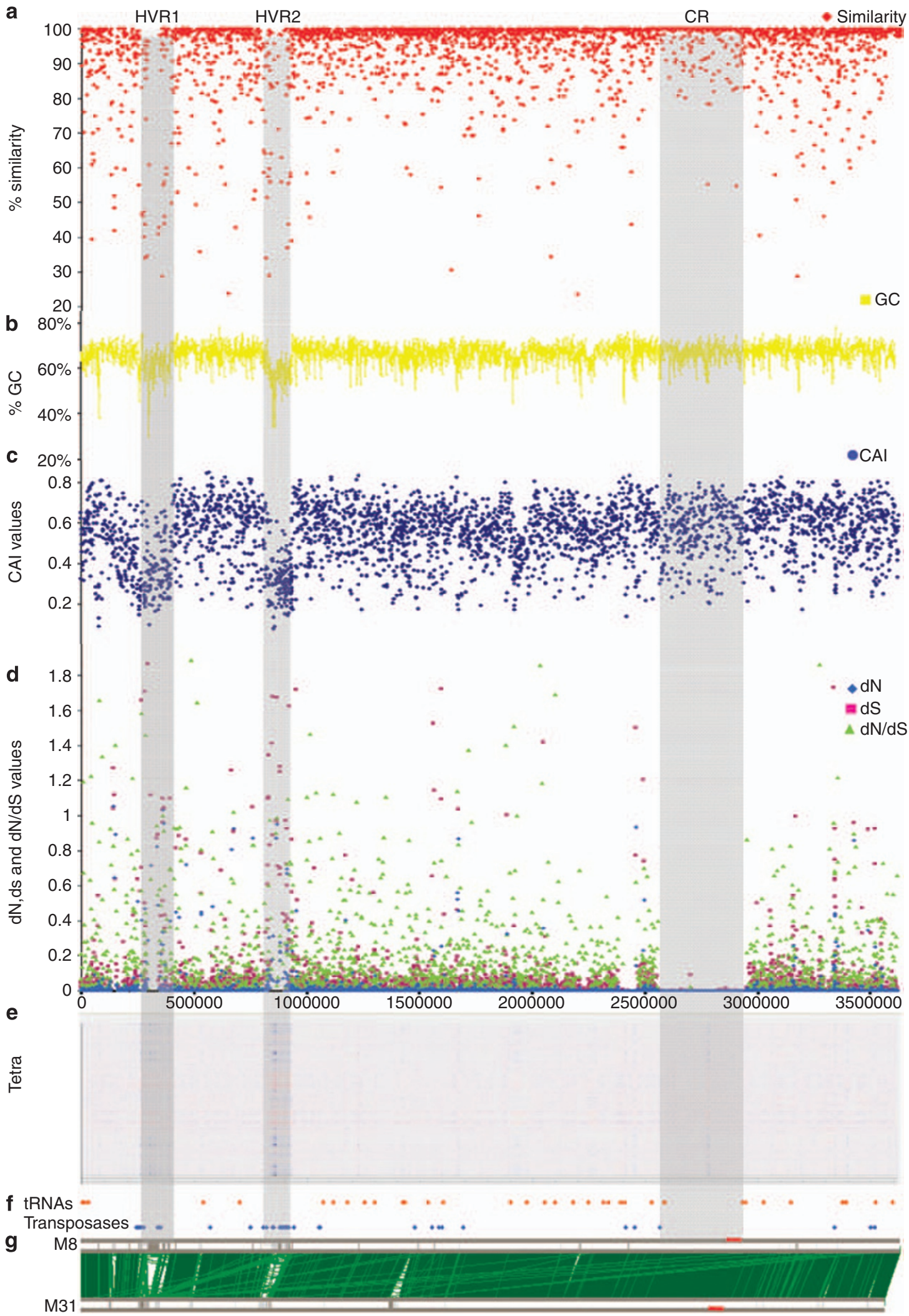

Figure 2 Summary and data comparison of S. ruber M8 chromosome. (a) Identities to M31 orthologous genes. (b) G+C content. (c) CAI indexes. (d) dN, dS and dN/dS values for homologous. (e) TETRA (tetranucleotide usage patterns in DNA sequences). (f) Transfer RNAs (tRNAs) and transposases along the chromosome. (g) Whole-genome alignment between S. ruber M8 and M31.The sequences have been aligned from the predicted replication origin. The green bars linking both genomes represent ortholog matches identified by FASTA (fast nucleotide comparison) analysis with a 100 nucleotide window. 


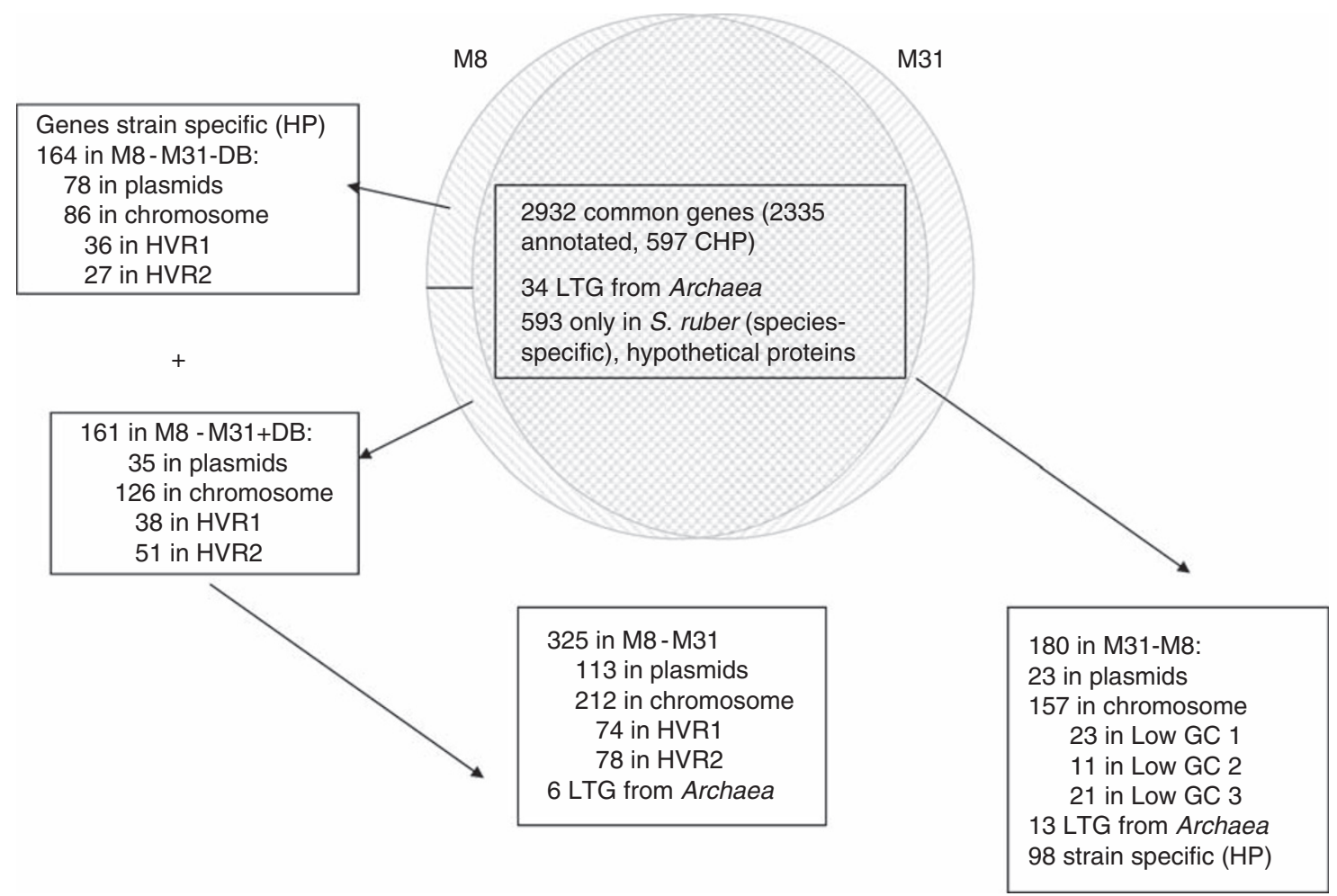

Figure 3 Overall comparison of M8 and M31.

than those based on the comparison of the full sequences (ANIb). The latter yields results that are more comparable to those obtained by DNA-DNA hybridization experiments. Low values of amino acid identities have also been reported for distinct ecotype representatives within other species such as Prochlorococcus spp. (Coleman et al., 2006) and A. macleodii (Ivars-Martínez et al., 2008).

Phylogenetic reconstructions revealed 20 gene duplications in the M8 genome that were absent from M31, and that likely occurred after the divergence of both strains. As many as 14 of these duplications corresponded to genes encoding putative transposases. Transposase duplication is sometimes associated with IS (insertion sequences) transposition events, although for most cases we did not detect other typical IS features such as terminal inverted repeats or flanking direct repeats.

The average non-synonymous (dN) to synonymous (dS) substitution rates for $S$. ruber genes was 0.125. This value lies between that of Escherichia coli (0.081) and that reported for pathogens inhabiting a narrow range of niches such as Helicobacter pylori (0.188) and Neisseria meningitidis (0.158) (Jordan et al., 2002). The low value in E. coli is considered to be related to an enhanced purifying selection caused by its large population size (Jordan et al., 2002). S. ruber would be an intermediate case, because its population sizes are remarkably high, but it does not seem to undergo dramatic niche changes. Forty-one genes had dN/dS values above one, which means that they are probably under positive selection (Jordan et al., 2002), and 25 of these are species-specific hypothetical proteins. A possible explanation for these high ratios is that the genes were acquired recently and are still in the process of adaptation to their new context. Unusually high $\mathrm{dN} / \mathrm{dS}$ ratios have also been observed in other closely related strains (Rocha et al., 2006) as a result of paralog formation, that is, recent gene duplications in which one gene undergoes a process of neo- or sub-functionalization.

Remarkable is the presence of a region (2 $583792-$ $2960363 \mathrm{bp}$, 'CR') with a high degree of sequence conservation $(99.5 \%)$ in which there were no nonsynonymous changes. In the cases in which the similarity among the predicted proteins was not $100 \%$, the differences were always caused by insertion or deletions within the genes. This region was enriched in genes of the clusters of orthologous groups categories $\mathrm{E}$ (amino acid transport and metabolism) and $\mathrm{P}$ (inorganic ion transport and metabolism), with most genes belonging to the transport rather than metabolism part of these categories. More specifically, all the CR-encoded proteins in clusters of orthologous group class $\mathrm{P}$ were inorganic ion transporters (mostly for potassium, iron and phosphate). In addition, eight out of nine amino acid transporters that were annotated in the genome were located in the CR. In good agreement, rapid annotations using subsystems technology analyses (Aziz et al., 2008) indicated that genes 


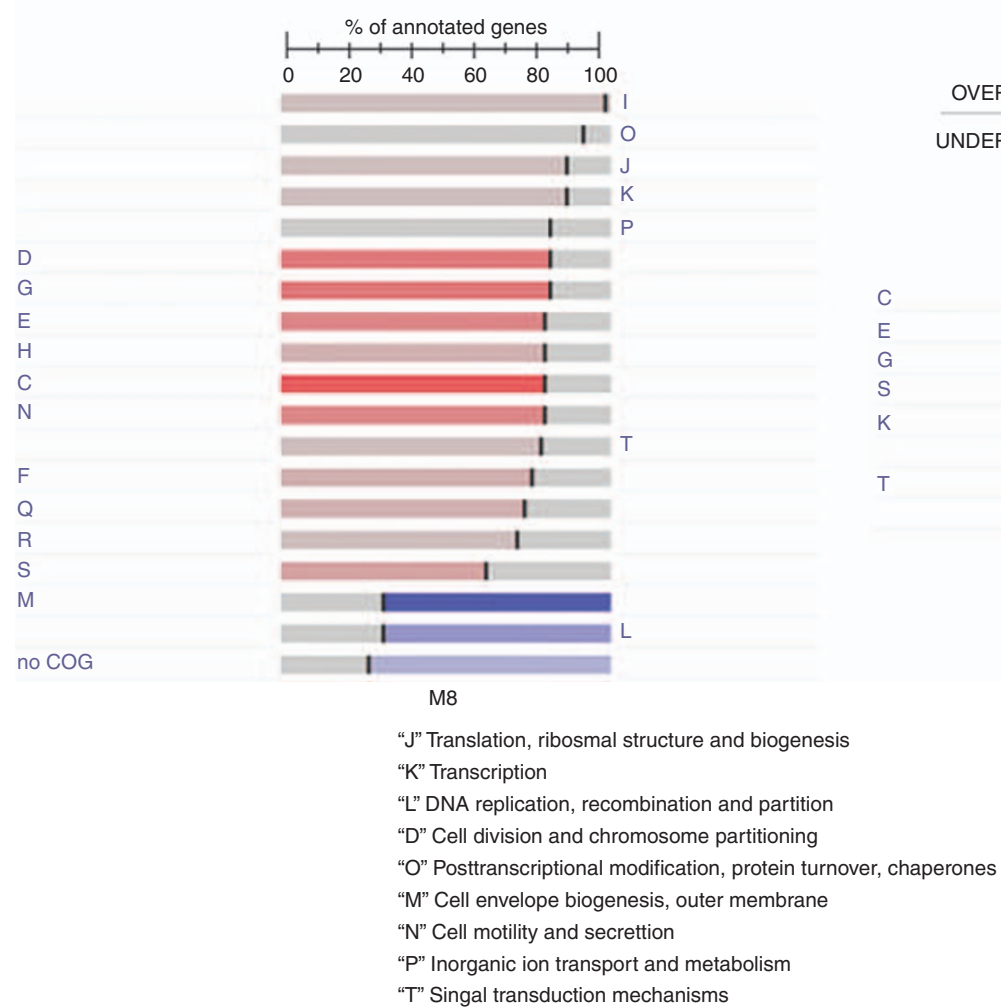

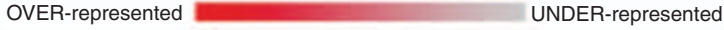

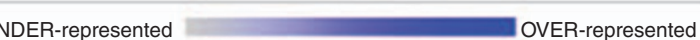

$\%$ of annotated genes

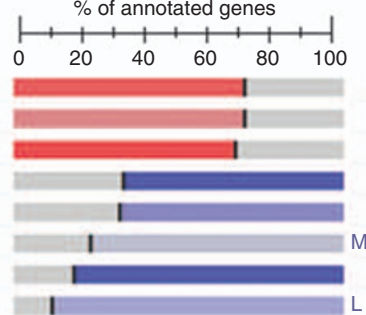

M31

"C" Energy production and conversion

"G" Carbohydrate transport and metabolism

"E" Amino acid transport and metabolism

"F" Nucleotide transport and metabolism

"H" Coenzyme metabolism

"l" Lipid metabolims

"Q" Secondary metabolites biosynthesis, transport and catabolism

"R" General functional prediction only

"S" Function unknown

Figure 4 Over- and underrepresented clusters of orthologous group (COG) functional classes in M8 and M31 genes ranked according their codon usage. Classes with red bars are over-represented among the genes with lower CAI value, while classes with blue bars aver over-represented among genes with higher CAI values. Over- and underrepresentation was estimated by a segmentation test as implemented in the Fatiscan program.

of the subsystems 'potassium metabolism', 'membrane transport' and 'phosphorus metabolism' were highly enriched in the CR. These zone only contained genes present in both strains, including the transporters encoded in the 'halophilic island' that has previously been reported for $S$. ruber M31 (Mongodin et al., 2005).

Codon usage indexes were rather constant along $S$. ruber genome, with the exception of HVRs in which genes with poorer indexes were concentrated. With only one exception, the genes with the $10 \%$ highest codon adaptation indexes (CAIs, a measure of codon usage) (above 0.716), were common to M8 and M31. However, M8 and M31 showed also small differences in codon usage (Figure 4). It is noteworthy that many genes involved in transport as well as in energy and nucleotide metabolism show the highest CAIs, while genes with low indices are mostly hypothetical proteins and transposases. A majority of the ribosomal proteins had an average CAI, which is typical for many slow-growing microorganisms (Carbone et al., 2003) and thus applies to most environmental bacteria.

Hypervariable regions or genomic islands. Two large zones of anomalously low $\mathrm{G}+\mathrm{C}$ content, poorer CAIs and deviant tetranucleotide signatures were identified in the chromosome of M8 at positions 0.25 and $0.82 \mathrm{Mb}$ from the origin of replication. We will refer to these regions as the hypervariable regions HVR1 and HVR2 (as in Whilhelm et al. (2007)). HVRs corresponded (see Figure 1, bottom and Supplementary Figure 2) with two of the three genomic islands previously described in $S$. ruber M31 (Mongodin et al., 2005) and could thus represent islands of high genomic variability in this species. A general terminology for hypervariable regions does not seem to exist, however, they are often referred to as 'islands' in analogy to pathogenicity islands, although genomic islands are usually considered to be mobile genetic elements (Dobrindt et al., 2004). Genomic islands have been reported for many prokaryotes, ranging from human pathogens to free-living marine bacteria or extremely halophilic Archaea. However, in contrast to most of the regions described so far (Dobrindt et al., 2004), HVRs in $S$. ruber M8 were clearly not associated with transfer RNAs (Figure 1). Such a lack of transfer RNA association has also been found for metagenomic islands detected in the environmental metagenome of the extremely halophilic 
Table 2 General characteristics of the HVRs presents in S. ruber M8

\begin{tabular}{lcc}
\hline & HVR1 & HVR2 \\
\hline General characteristics & & \\
Average GC\% & 60.6 & 58.2 \\
Origin & 256919 & 830124 \\
End & 396085 & 956929 \\
Length (bp)/\% & $139166 / 3.8$ & $126845 / 3.5$ \\
Coding density \% & 75.5 & 59.84 \\
& & \\
Genes & Number/\% & Number/\% \\
& & \\
\hline M8-M31-DB & & \\
M8-M31+DB & & \\
M8+M31-DB & & $51 / 40.40$ \\
M8+M31+DB & $36 / 41.86$ & $14 / 2.56$ \\
Total: & $15 / 2.56$ & $59 / 2.58$ \\
Transposases & $42 / 1.83$ & $151 / 4.89$ \\
Glycosyltranferases & $131 / 4.25$ & $20 / 31.75$ \\
Sulfotransferases & $16 / 25.40$ & $3 / 1$ \\
COG M & $9 / 30$ & $2 / 25$ \\
Phage related & $3 / 37.5$ & $22 / 13.84$ \\
CHP & $13 / 8.18$ & $3 / 42.86$ \\
HP & $0 / 0$ & $61 / 5.11$ \\
& $41 / 3.43$ & $27 / 31.40$ \\
\hline A & $36 / 41.86$ & \\
\hline
\end{tabular}

Abbreviations: CHP, conserved hypothetical protein; COG, clusters of orthologous group; HP, hypothetical protein; HVRs, hypervariable regions.

aPercentage of the total genes in this category, in the chromosome. ${ }^{\mathrm{b}}$ Genes M8 specific, absent from M31 and the rest of the database.

${ }^{\mathrm{C}} \mathrm{Genes}$ present in M8 and some other organisms in the database but absent from M31.

${ }^{\mathrm{d}}$ Genes species specific, present only in M8 and M31.

${ }^{\mathrm{e}} \mathrm{Genes}$ present in M8, M31 and some other organism in the database.

archaeon $H$. walsbyi and was suggested as a characteristic of archaeal genomic islands (CuadrosOrellana et al., 2007). Annotation and functional classification revealed that $21 \%$ of the genes belonging to the clusters of orthologous group $M$ (cell envelope biogenesis, outer membrane) are located in HVRs, which account only for $7.7 \%$ of the chromosome. This difference in gene content is particularly pronounced within HVR2, for which rapid annotations using subsystems technology analyses (Aziz et al., 2008) only detected genes belonging to the subsystem 'cell wall and capsule'. Similarly, the presence of genes coding for transposases, glycosyltransferases, and sulfotransferases was found to be remarkably high in HVRs (Table 2).

When the M8 genome was used for contig recruitment of environmental clones from a solar saltern in San Diego, a lower coverage of HVR was found, indicating that these regions were underrepresented in the metagenome (Supplementary Figure 3). Comparisons of genomes from Prochlorococcus (Coleman et al., 2006), Haloquadratum (CuadrosOrellana et al., 2007) and Pelagibacter (Whilhelm et al., 2007) with corresponding metagenomes have provided similar results showing underrepresentation of HVRs in contig recruitment. Similarly, M8 strain-specific genes (Figure 2), extremely divergent orthologous genes (identities below 50\%) (Figure 2, blue lines in the forth circle inward), and genes whit higher dNs values are overrepresented in these zones. Combined codonw and CAIJAVA results (Supplementray Table 1), that can provide valuable hints on the expression levels of genes because a high degree of codon adaptation results in higher translational efficiencies and thus higher expression levels, and their visualization (Figure 2) revealed that many genes within the HVRs have low CAI values, which could be an indication of recent acquisition.

\section{Involvement of interdomain (Archaea-bacteria) LGT} in the shaping of $\mathrm{S}$. ruber genome

As $S$. ruber shares its habitat with extremely halophilic Archaea, in particular with $H$. walsbyi, it has been suggested as a good candidate for interdomain LGT events. This hypothesis was reinforced by the many phenotypic similarities found between $S$. ruber and many extremely halophilic Archaea (Antón et al., 2008). The analysis of the S. ruber M31 genome suggested that this was indeed the case, although the number of genes likely involved in LGT was found to be lower than initially anticipated (Mongodin et al., 2005). To increase the stringency of these analyses, we used a two-step approach to identify genes likely transferred to the M8 genome from Archaea (or the other way around). In the first step, we identified 40 candidate genes for interdomain LGT (Supplementary Table 2) by sequence comparison and phylogenetic analysis, which were then scrutinized by additional methods in the second step. A majority of these genes coded for membrane proteins, including retinal-binding proteins, adaptive-response sensory kinases and ion uptake proteins. Moreover, 6 of the 40 LGT candidates were strain-specific for $S$. ruber M8 and, conversely, 13 of the 47 genes that were reported earlier as inter-domain LGT candidates in S. ruber M31 (Mongodin et al., 2005) lacked any homolog in S. ruber M8.

We subsequently analyzed LGT candidates using self-organizing maps and also compared the di, tri- and tetranucleotide frequencies found in the 40 LGT candidate genes to the corresponding frequencies within 572 prokaryote genomes and plasmids (Supplementary Table 2). Many of them exhibited weak similarities to halophilic Archaea, most pronouncedly reflected in their dinucleotide signature. However, for the majority of the genes the results lacked consistency over all oligonucleotides and therefore the derived classification was ambiguous. In some cases, $S$. ruber oligonucleotide frequencies also showed good correlations to those of Actinobacteria, most likely a result of their common high $\mathrm{G}+\mathrm{C}$ contents. The results of the self-organizing map-classification are summarized in Supplementary Table 2. A majority of the LGT candidates were classified to $S$. ruber and only eight LGT candidates were found to have a clear archaeal signal according to this procedure. Three of these 
genes (Supplementary Table 2) were present in $S$. ruber M8 but not in $S$. ruber M31.

As the presence of genes with an atypical codon composition is a strong indicator of LGT (Abby and Daubin, 2007), we performed a codon usage analysis of the LGT candidates. Codon usage analysis of the LTG candidates revealed average to good codon usage adaptation indices for most of the LGT candidates present in both strains. This might be an indication that in these cases, the LGT was not recent and the codon usage of these genes has been adapted to the $S$. ruber host genome. Consistently, LGT candidates present in M8 but not in M31 had rather poor codon usage indices, suggesting more recent LGT events, most likely after the separation of the M8 and M31 lineages.

Reverse transcriptase-PCR analyses indicated that 31 of the 34 inter-domain LGT candidates present in both strains were transcribed at some point of the growth curve, although the expression patterns of some genes showed slight differences between the two strains. The residual three genes were not expressed in any strain under any of the conditions analyzed. Under standard conditions, expression could only be detected for three out of the six S. ruber M8-specific inter-domain LGT candidates (Supplementary Table 2, blue color). Similarly, only one of the eight $S$. ruber M31-specific inter-domain LGT candidates analyzed was found to be expressed under standard growth conditions. Overall, these results indicate that most of the species-specific inter-domain LGT candidates represent functional genes and are expressed during normal growth under laboratory conditions, although for the strain-specific inter-domain LGT candidates this is only the case for a small fraction.

Thus, besides being constitutively transcribed, most LGT candidates are not clustered in HVRs, instead they constitute part of the (so far) core genome of the species, including the halophilism island present in CR. In a way, LGT from Archaea, although not very extensive, has had a role in the shaping of $S$. ruber species.

\section{Metabolomic comparisons of M8 and M31}

As one of the most apparent differences in HVRs is the abundance of genes coding for sulfotransferases and glycosyltransferases, the metabolomes of M8 and M31 were compared for the presence of sulfonated/ S-containing and glycosylated metabolites.

FT-ICMS comparison of signal pairs at exact mass differences corresponding to sulfonation and glycosylation indicated a consistent increase in sulfonated and glycosylated metabolites in M8 compared with M31 and this increase was considerably higher in the extracellular fraction of the cultures (Table 3). In addition, the exact masses obtained with electrospray ionization (-)-ultrahigh resolution mass spectrometry were converted into more than 2000 elementary compositions (C, H, O, N and S).
Table 3 Proportion of sulfonated and glycoylated metabolites in M8 and M31

\begin{tabular}{lcc}
\hline & \multicolumn{2}{c}{ Salinibacter ruber } \\
\cline { 2 - 3 } Metabolites & M8 & M31 DSM 13588 \\
\hline All fractions & & \\
$\quad$ Sulfonated & 1.7 & 1.5 \\
$\quad$ Glycosylated & 4.4 & 3.1 \\
Extracellular fraction & & \\
$\quad$ Sulfonated & 2.7 & 1.8 \\
Glycosylated & 6.1 & 2.6 \\
\hline
\end{tabular}

${ }^{a}$ Intracellular soluble, pellets and extracellular (see Materials and methods).

These analyses also supported a significant increase of sulfur containing metabolites mainly in the extracellular fraction of M8 versus M31 from 21.8\% to $28.5 \%$ relative to all calculated $\mathrm{CHO}$ (Carbon, Hydrogen, Oxygen) type of molecules.

Finally, the exact mass lists were assigned to specific metabolites of $S$. ruber within the KEGG database using the MassTRIX annotation interface (Suhre and Schmitt-Kopplin, 2008, www.masstrix. org); with this approach only $15-25 \%$ of the masses are assigned to metabolites in possible pathways. Small differences were observed between M31 and M8 metabolomes. However, cytoplasmic and extracellular fractions of M8 contained more metabolites related to pathways involving amino acids and carbohydrates/fatty acids, than the equivalent fractions in M31. When the Japanese Metabolome database (www.metabolome.jp) was used for metabolite annotation, a significant increase to more than $26 \%$ was found with respect to the number of S-containing molecules in the extracellular M8 fraction, such as hexose-sulfates, gluthathiones (see Figure 5), sulfobenzoate or sulfobenzaldehyde. When comparing for glycosylated metabolites, a systematically higher number of hits was found also for M8 when compared with M31. Regardless of the database used for metabolite annotation, the proportion of hits within supernatant samples to glycosylated metabolites relative to all hits is significant in $\mathrm{M} 8,(+10 \%$ to $+60 \%$ from the Japanese database and from $+10 \%$ to $+180 \%$ from the MassTRIX approach for cytoplasmic and extracelular fractions respectively).

Thus, the main metabolomic differences detected by all approaches in M8 relative to M31 are related to molecules released to the medium or loosely attached to the cell surface that could have been released during the sample processing.

\section{Phage susceptibility}

Many of the M8 and M31 (Mongodin et al., 2005) genes in HVRs were related to cell-surface properties that could have a role in phage recognition and evasion. Metabolomic analyses also indicated 


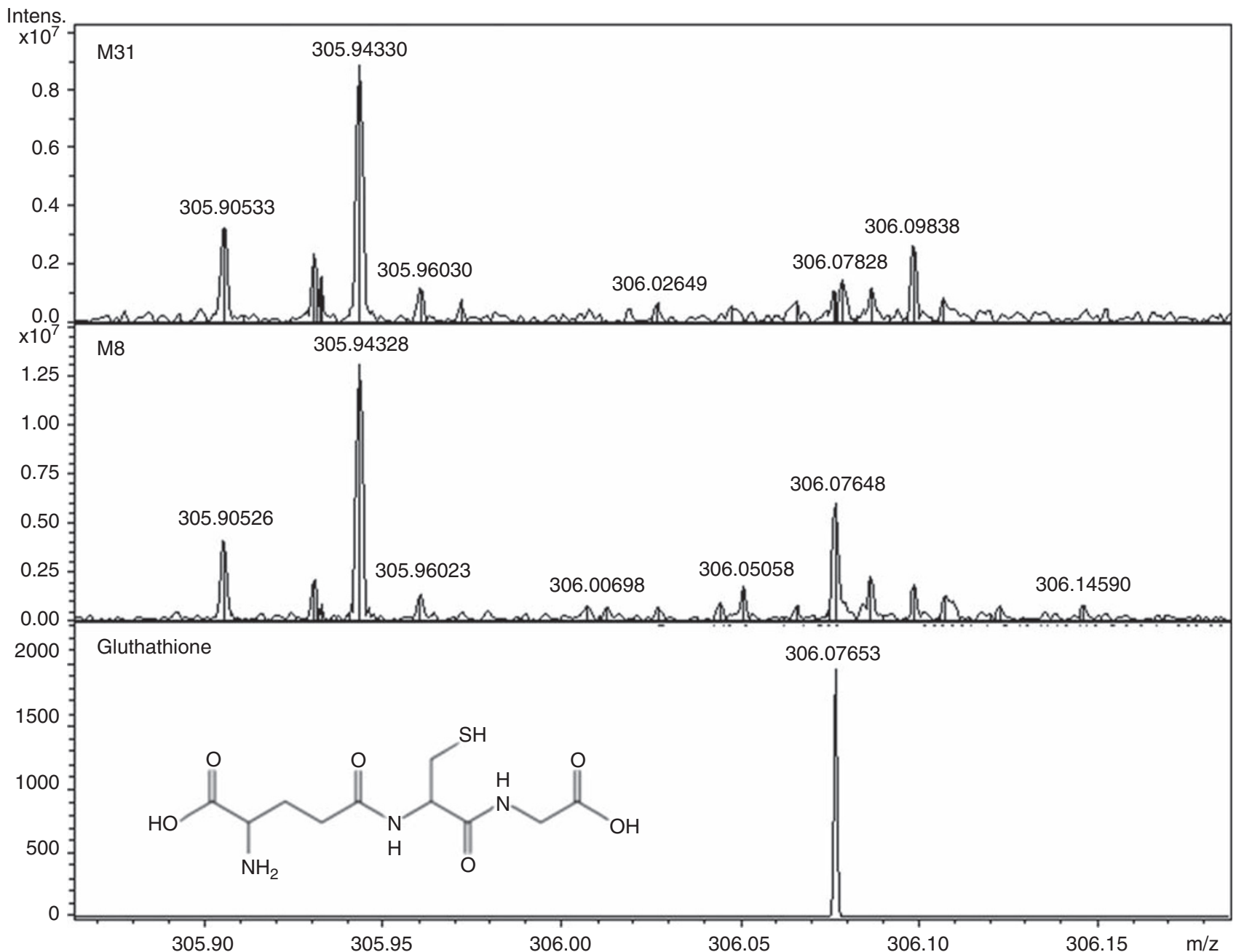

Figure 5 Example of relative increase in ion (C10H16O6N3S)—in supernatant of M8 relative to M31, corresponding to the elementary composition of gluthathione (simulated spectra).

differences in the exposed components of M8 and M31. Hypersaline environments show one of the highest numbers of viruses reported for planktonic systems with values of up to $2 \times 10^{9}$ virus-like particles per $\mathrm{ml}$ in crystallizers from which Salinibacter representatives have been isolated (Guixa-Boixareu et al., 1996). Halophages are thus considered to be important agents in mortality in hypersaline environments, given that no bacteriovory can be detected at salinities above 25\% (Guixa-Boixareu et al., 1996). Therefore, cell-surface properties are likely under strong selection, as suggested for the extremely halophilic archaeon $H$. walsbyi (CuadrosOrellana et al., 2007), and for marine bacteria that are exposed to high viral predation, for example, Prochlorococcus (Coleman et al., 2006) or Pelagibacter representatives (Whilhelm et al., 2007) for which surface-related proteins have also been observed in genomic island(s) or hypervariable regions within their genomes. Phages thus seem to have a high effect in driving micro-diversification within prokaryote species (Rodríguez-Valera et al., 2009), not only because of their direct selective pressure on exposed cellular components but also because of their role in LGT processes. Besides cellsurface manipulation and exopolysaccharide production, Bacteria and Archaea have other viral defense mechanisms such as the CRISPR (clustered regularly interspaced short palindromic repeats) system that has been proved to confer resistance to phage infection in Streptococcus thermophilus and, according to a wealth of metagenomic data, are considered to be a general mechanism of phage defense (Wilmes et al., 2008). However, S. ruber M8 and M31 lack this system.

According to the genomic and metabolomic data, it should be expected than strains M8 and M31 would show different phage susceptibility. Indeed, when we first tried to isolate $S$. ruber phages from Mallorca sample waters, we only succeeded in getting phages from M31, while not a single phage from M8 could be obtained. To quantify the different levels of phage susceptibility in both strains, we 
infected cultures with virus assemblages from two different water samples (salt concentrations of $23.2 \%$ and $34.2 \%$, see Materials and methods) obtained from Santa Pola salterns. For the lower salt water, M8 had plaque counts of $4.47 \times 10^{2} \pm 61.1 \mathrm{PFU} \mathrm{ml}^{-1}$ of natural sample and M31, $6.33 \times 10^{2} \pm 65.1 \mathrm{PFU} \mathrm{ml}^{-1}$. However, for the high salt sample, M8 had a higher plaque count $\left(1.6 \times 10^{4} \pm 3.61 \times 10^{3} \mathrm{PFU} \mathrm{ml}^{-1}\right)$ while no plaques were formed by M31 under the assayed conditions.

\section{Competition between strains M8 and M31 under saturated salt conditions}

The observation of metabolome differences between $S$. ruber strains M8 and M31 prompted us to analyze whether one of the two strains would have a growth advantage over the other under certain conditions. Thus, we set up competition experiments in which mixed cultures of $S$. ruber M8 and M31 were compared with pure cultures of each strain. Growth was monitored by OD (optical density)-reading and 4',6-diamidino-2-phenylindole (DAPI) counting, and in mixed cultures cell numbers for M8 and M31 were determined also by quantitative PCR (see Supplementary Material). We tested for possible competition between the two strains under standard growth conditions and under salt-saturated conditions as they occur also in the natural habitat because crystallizer ponds are frequently saturated in $\mathrm{NaCl}$. Under standard growth conditions, $S$. ruber M8 outcompeted S. ruber M31 (Figure 6a). However, in salt-saturated medium (Figure 6b), the density of $S$. ruber M31 in mixed cultures was roughly up to 30 -fold higher than that of $S$. ruber M8, although in pure cultures the difference was only twofold. This indicates that under saturated salt conditions $S$. ruber M31 hinders growth of $S$. ruber M8, which in turn strongly suggests direct competition between the two closely related populations also in situ. Indeed, the salterns are periodically submitted to cycles of refilling and emptying, and thus Salinibacter spp. are exposed to fluctuating salt concentrations. This does not necessarily mean that these two strains are actually competing in nature, because dilution of every genotype could be too high to allow for the competition between them, such as reported for V. splendidus (Thomson et al., 2005). As pointed out by Wilmes et al. (2008), 'the set of variable genes and genome rearrangements may be so large in some populations that no individuals have exactly the same genotype'. In the case of $S$. ruber, our previous studies indicate a high degree of genomic diversity between different isolates from the same environment (unpublished results) although, in any case, we show here that there is a real possibility of competition of extremely closely related strains. Then, given the appropriate selective force, one strain could displace the other and thus give raise to sympatric differentiation and proceed to broader genomic differences.
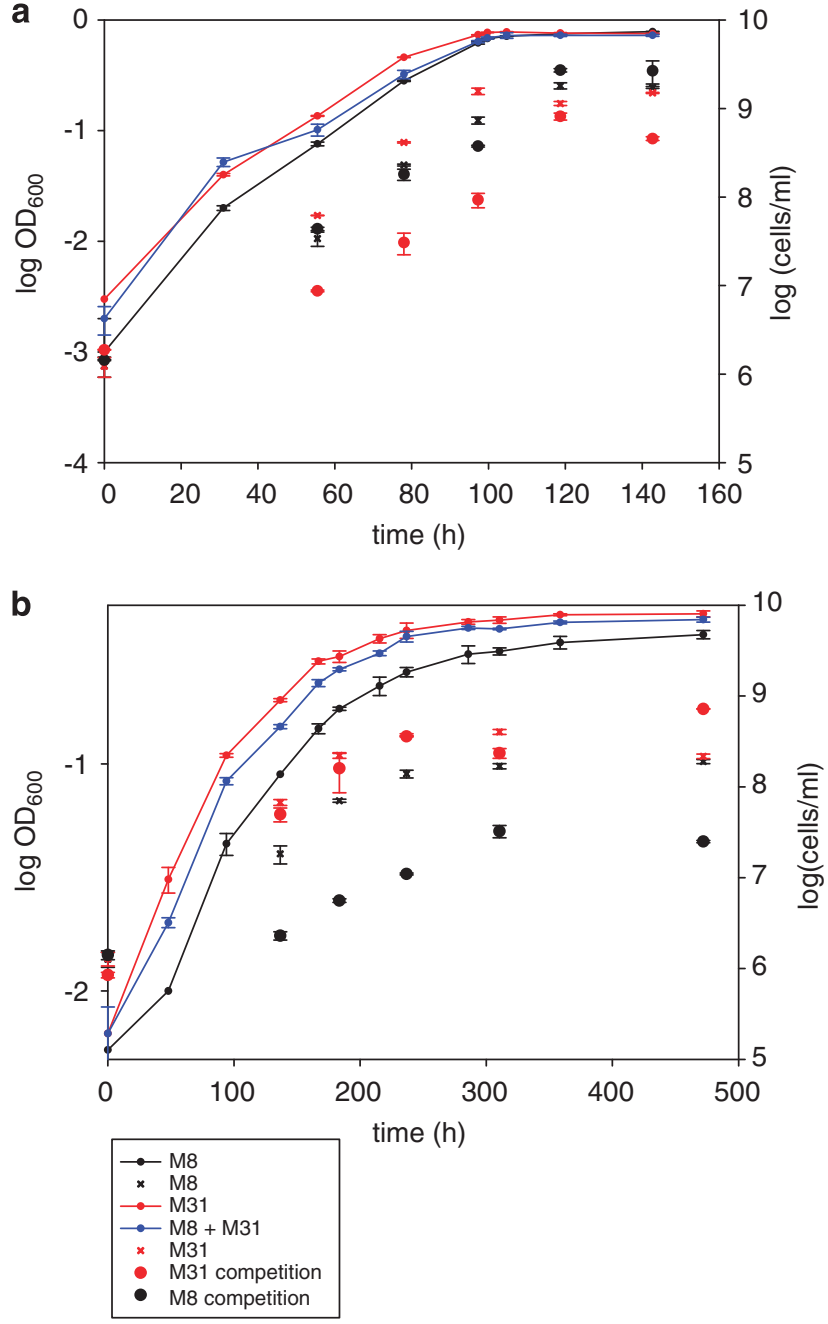

Figure 6 Competition experiments between strains M8 and M31 grown under standard conditions (a) and in NaCl-saturated medium (b). Lines represent OD reads for pure and mixed cultures, as indicated in the insert. For mixed culture, the values of M8 and M31 measured by quantitative PCR are represented by black and red dots, respectively. Crosses represent the values of M8 (black) and M31 (red) measured by fluorescence in situ hybridization (FISH) in pure cultures of every strain.

These changes in $S$. ruber strain abundances could never have been detected in nature by means of the commonly used rRNA-based standard tools of molecular ecology. Certainly, 16S rRNA genes are very conserved, and thus not useful to unveiling micro-diversity. However, ITS are far less conserved and frequently used for assessing coexisting populations of the same species in situ (for example, Prochlorococcus, (Coleman et al., 2006)). In addition, many micro-diversity landscapes have been described in terms of rRNA diversity (Acinas et al., 2004; Pedrós-Alió, 2006). Thus, the use of rRNA/ITS methods to detect population dynamics in natural samples provides only a partial picture because it can overlook ecological relevant changes in microdiversity within a species. 


\section{Conclusions}

Speciation is the generation of permanently distinct clusters of closely related bacteria (Fraser et al., 2007). M8 and M31 are genetically distinct strains that, as shown by the competition and phage infection experiments, can respond differently to external conditions and could thus undergo sympatric differentiation in their environment. Whether differences between M8 and M31 are neutral or not depends more on the environment than on the genomes themselves, as reported for Vibrio and Roseobacter members (Polz et al., 2006) that had differences adaptive under ecological circumstances and neutral under another. Although M31 could displace M8 under the appropriate circumstances, this may not be occurring in nature because competition can be part of a dynamic process, with a succession of different genotypes of the population that change their concentrations with time, originating a reservoir of genetic diversity inside a sequence space that would constitute the species $S$. ruber. We have captured the evolution of this species in the motion but the direction to which it will proceed cannot be predicted. To clarify this point, it would be necessary to study the changes within the M8/M31 genomes over time in their true ecological context, which includes not only habitat resources and physical conditions but also phages and predators as well as the substances secreted by other organisms (Cohan and Koeppel 2008).

Beyond the controversy of how many microbial species exist (Pedrós-Alió, 2006), or even if they exist at all (Doolittle and Zhaxybayeva, 2009), a new 'unknown' is rising regarding the functional diversity and environmental adaptation of bacteria. This study is showing the extent of genome, phenotype and niche differentiation encoded by rather small inter-strain differences, a phenomenon that most likely is very widespread in the environment.

\section{Acknowledgements}

We warmly thank Carlos Pedrós-Alió and Johaness Sikorski for the critical reading of a previous version of the paper and their many helpful suggestions and discussion. This work was funded by projects CGL200612714-CO2-01 and 02 from de Spanish Ministry of Science (to JA and RRM). J Dopazo thanks the National Institute of Bioinformatics (www.inab.org), that is a platform of Genoma España. This work was financially supported by the Max Plank Society within the projects Marine Genomics and EnviTools.

\section{References}

Abby S, Daubin V. (2007). Comparative genomics and the evolution of prokaryotes. Trends Microbiol 15: 135-141.

Acinas SG, Klepac-Ceraj V, Hunt DE, Pharino C, Ceraj I, Distel DL et al. (2004). Fine-scale phylogenetic architecture of a complex bacterial community. Nature 430: $551-554$.

Antón J, Oren A, Benlloch S, Rodríguez-Valera F, Amann R, Rosselló-Mora R. (2002). Salinibacter ruber gen. nov., sp. nov., a novel, extremely halophilic member of the Bacteria from saltern crystallizer ponds. Int J Syst Evol Microbiol 52: 485-491.

Antón J, Peña A, Santos F, Martínez-García M, SchmittKopplin P, Rosselló-Mora R. (2008). Distribution, abundance and diversity of the extremely halophilic bacterium Salinibacter ruber. Saline Syst 4: 1-10.

Aziz RK, Bartels D, Best AA, DeJongh M, Disz T, Edwards RA et al. (2008). The RAST server: rapid annotations using subsystems technology. BMC Genomics 9: 75.

Carbone A, Zinovyev A, Kepes F. (2003). Codon adaptation index as a measure of dominating codon bias. Bioinformatics 19: 2005-2015.

Cohan FM, Koeppel AF. (2008). The origins of ecological diversity in prokaryotes. Curr Biol 18: R1024-R1034.

Coleman ML, Sullivan MB, Martiny AC, Steglich C, Barry $\mathrm{K}$, DeLong EF et al. (2006). Genomic islands and the ecology and evolution of Prochlorococcus. Science 311: 1768-1770.

Cuadros-Orellana S, Martín-Cuadrado AB, Legault B, D'Auria G, Zhaxybayeva O, Papke RT et al. (2007). Genomic plasticity in prokaryotes: the case of the square haloarchaeon. ISME J 1: 235-245.

Dobrindt U, Hochhut B, Hentschel U, Hacker J. (2004). Genomic islands in pathogenic and environmental microorganisms. Nat Rev Micro 2: 414-424.

Doolittle WF, Zhaxybayeva O. (2009). On the origin of prokaryotic species. Genome Res 19: 744-756.

Feil EJ. (2004). Small change: keeping pace with microevolution. Nat Rev Microbiol 2: 483-495.

Fleischmann RD, Adams MD, White O, Clayton RA, Kirkness AR, Bult CJ et al. (1995). Whole-genome random sequencing and assembly of Haemophilus influenzae Rd. Science 269: 496-512.

Fraser C, Hanage WP, Spratt BG. (2007). Recombination and the nature of bacterial speciation. Science $\mathbf{3 1 5}$ : 476-480.

Guixa-Boixareu N, Calderón-Paz JI, Heldal M, Bratbak G, Pedrós-Alió C. (1996). Viral lysis and bacterivory as prokaryotic loss factors along a salinity gradient. Aquat Microb Ecol 11: 215-227.

Huerta-Cepas J, Bueno A, Dopazo J, Gabaldón T. (2008). PhylomeDB: a database for genome-wide collections of genes phylogenies. Nucl Acids Res 36: D491-D496.

Hunt ED, David LA, Gevers D, Preheim SP, Alm EJ, Polz MF. (2008). Resource partitioning and sympatric differentiation among closely related bacterioplankton. Science 320: 1081-1085.

Huson DH, Bryant D. (2006). Application of phylogenetic networks in evolutionary studies. Mol Biol Evol 23: 254-267.

Ivars-Martínez E, Martín-Cuadrado AB, D’Auria G, Mira A, Ferriera S, Johnson J et al. (2008). Comparative genomics of two ecotypes of the marine planktonic copiotroph Alteromonas macleodii suggests alternative lifestyles associated with different kinds of particulate organic matter. ISME J 2: 1194-1212.

Jordan IK, Rogozin IB, Wolf YI, Koonin EV. (2002). Microevolutionary genomics of bacteria. Theor Popul Biol 61: 435-447.

Meddini D, Donati C, Tettelin H, Masignani V, Rappuoli R. (2005). The microbial pan-genome. Curr Opin Genet Dev 15: 589-594. 
Mongodin EF, Nelson KE, Daugherty S, DeBoy RT, Wister J, Khouri $\mathrm{H}$ et al. (2005). The genome of Salinibacter ruber: convergence and gene exchange among hyperhalophilic bacteria and archaea. Proc Natl Acad Sci USA 102: 18147-18152.

Papke RT, Koening JE, Rodríguez-Valera F, Doolittle WF. (2004). Frequent recombination in a saltern population of Halorubrum. Science 306: 1928-1929.

Pedrós-Alió C. (2006). Marine microbial diversity: can it be determined? Trends Microbiol 14: 257-263.

Peña A, Valens-Vadell M, Santos F, Buczolits S, Antón J, Kämpfer $\mathrm{P}$ et al. (2005). Intraspecific comparative analysis of the species Salinibacter ruber. Extremophiles 9: 151-161.

Polz MF, Hunt DE, Preheim SP, Weinreich DM. (2006). Patterns and mechanisms of genetic and phenotypic differentiation in marine microbes. Phil Trans $R$ Soc $B$ 361: 2009-2021.

Rocha EPC, Smith JM, Hurst LD, Holden MTG, Cooper JE, Smith $\mathrm{NH}$ et al. (2006). Comparisons of $\mathrm{dN} / \mathrm{dS}$ are time dependent for closely related bacterial genomes. J Theor Biol 239: 226-235.

Rodríguez-Valera F, Martín-Cuadrado AB, Rodríguez-Brito B, Pasić L, Thingstad TF, Rohwer F et al. (2009). Explaining microbial population genomics through phage predation. Nat Rev Microbiol 7: 828-836.

Rosselló-Mora R, Lucio M, Peña A, Brito-Echeverría J, López-López A, Valens-Vadell M et al. (2008). Metabolic evidence for biogeographic isolation of the extremophilic bacterium Salinibacter ruber. ISME J 2: 242-253.

Sikorski J. (2008). Populations under microevolutionary scrutiny: what will we gain? Arch Microbiol 189: 1-15.

Suhre K, Schmitt-Kopplin P. (2008). MassTRIX: mass translator into pathways. Nucl Acids Res 36: W481-W484.

Thomson JR, Pacocha S, Pharino C, Klepac-Ceraj V, Hunt DE, Benoit J et al. (2005). Genotypic diversity within a natural coastal bacterioplankton population. Science 307: 1311-1313.

Whilhelm LJ, Tripp HJ, Givan SC, Smith DP, Giovannoni SJ. (2007). Natural variation in SAR11 marine bacterioplankton genomes inferred from metagenomic data. Biol Direct 2: 19.

Wilmes P, Simmons SL, Denev VJ, Banfield JF. (2008). The dynamic genetic repertoire of microbial communities. FEMS Microbiol Rev 33: 109-132.

Supplementary Information accompanies the paper on The ISME Journal website (http://www.nature.com/ismej) 